Cite this: Phys. Chem Chem. Phys. 2017, 19, 9409

Received 15th February 2017 Accepted 3rd March 2017

DOI: $10.1039 / c 7 c p 01023 k$

rsc.li/pccp

\title{
Can charged colloidal particles increase the thermoelectric energy conversion efficiency? $\dagger$
}

\author{
Thomas J. Salez, ${ }^{a b}$ Bo Tao Huang, ${ }^{a}$ Maud Rietjens, ${ }^{a}$ Marco Bonetti, ${ }^{a}$ Cécile Wiertel- \\ Gasquet, ${ }^{a}$ Michel Roger, ${ }^{a}$ Cleber Lopes Filomeno, ${ }^{c d}$ Emmanuelle Dubois, ${ }^{c}$ \\ Régine Perzynskic and Sawako Nakamae*a
}

\begin{abstract}
Currently, liquid thermocells are receiving increasing attention as an inexpensive alternative to conventional solid-state thermoelectrics for low-grade waste heat recovery applications. Here we present a novel path to increase the Seebeck coefficient of liquid thermoelectric materials using charged colloidal suspensions; namely, ionically stabilized magnetic nanoparticles (ferrofluids) dispersed in aqueous potassium ferro-/ferricyanide electrolytes. The dependency of thermoelectric potential on experimental parameters such as nanoparticle concentration and types of solute ions (lithium citrate and tetrabutylammonium citrate) is examined to reveal the relative contributions from the thermogalvanic potential of redox couples and the entropy of transfer of nanoparticles and ions. The results show that under specific ionic conditions, the inclusion of magnetic nanoparticles can lead to an enhancement of the ferrofluid's initial Seebeck coefficient by $15 \%$ (at a nanoparticle volume fraction of $\sim 1 \%$ ). Based on these observations, some practical directions are given on which ionic and colloidal parameters to adjust for improving the Seebeck coefficients of liquid thermoelectric materials.
\end{abstract}

\section{Introduction}

With no sign of slowing down in the global energy consumption, renewal energy science has become one of the highest research priorities worldwide. In this respect, low grade 'waste-heat' (e.g., from industrial waste streams and car exhausts) is regarded as a promising green energy source, albeit at smaller scales compared to other renewable sources such as solar, hydro, wind and biomass. One of the most straightforward routes to convert waste-heat into a more useful form of energy is to use 'thermoelectric' devices. Thermoelectric effects exist in many solid and liquid materials, but the most efficient thermoelectric converters (generator or coolers) today are all based on low-gap semiconductors. ${ }^{1-5}$ Complementary to the solid thermoelectric technologies, liquid-based thermoelectrochemical cells, or simply, thermocells are attracting increasing attention as a

\footnotetext{
${ }^{a}$ Service de Physique de L'État Condensé, SPEC, CEA, CNRS,

Université Paris-Saclay, CEA Saclay, 91191 Gif-sur-Yvette Cedex, France.

E-mail: sawako.nakamae@cea.fr

${ }^{b}$ École des Ponts ParisTech, 6 et 8 avenue Blaise Pascal, Champs-sur-Marne, F-77455 Marne-la-Vallée, France

${ }^{c}$ Laboratoire Physicochimie des Electrolytes et Nanosystèmes interfaciaux, UMR CNRS 8234, Université Pierre et Marie Curie - Paris 6, 4 place Jussieu, F-75009 Paris 5, France

${ }^{d}$ Grupo de Fluidos Complexos - Instituto de Quimica, Universidade de Brasilia, CP 04478, 70904-970 Brasilia (DF), Brazil

$\dagger$ Electronic supplementary information (ESI) available. See DOI: 10.1039/c7cp01023k
}

cheap and scalable alternative. ${ }^{6-9}$ Thermocells produce an electrical current through redox reactions when two electrodes are maintained at different temperatures. To enhance the thermocell performance (e.g., higher thermoelectric coefficient and larger electrical conductivity) various improvements are made through electrode materials, redox-couples, and electrolyte types, as well as the natural convection of liquids and the diffusion of dissolved ionic species..$^{8,10,11,12,13}$ So far, the highest power output reaching over $10 \mathrm{~W} \mathrm{~m}^{-2}$ has been reported very recently by Zhang et al. ${ }^{14}$ using a highly concentrated ferri-/ferrocyanide electrolyte.

In a thermocell containing nano- and micro-meter sized charged colloidal particles, the thermal drift of charged species can further add (or reduce) its total thermoelectric potential. The additional thermoelectric contribution, first introduced by Eastman ${ }^{15}$ and formalized more rigorously starting with Onsager's theory by De Groot ${ }^{16}$ and Agar, ${ }^{17}$ depends on the particles' effective charge, mobility and the Eastman entropy of transfer $\ddagger \hat{S}_{i}$. The latter is linked to the particle-particle as well as particle-solvent interaction nature. $\hat{S}_{i}$ is also closely related to the thermally induced concentration gradient known as the "Soret effect". 18-22 For relatively small ions, $\hat{S}_{i}$ 's are much smaller than the redox reaction entropy at electrodes and are often justifiably neglected

¥ The entropy of transfer considered here is the one derived by De Groot ${ }^{16}$ and Agar $^{17}$ from Onsager's theory. In this article, we will use Agar's terminology and refer it as "Eastman entropy of transfer." 
in typical thermocells. ${ }^{11,23,24}$ On the other hand, in colloidal solutions such as ferrofluids, very large $\hat{S}_{i}$ values have been measured $^{25}$ and have to be taken into account.

In order to determine the technological relevance of the thermo-electric diffusion to thermoelectric energy conversion, one must thus find a charged colloidal particle with sufficiently large Eastman entropy of transfer. Ferrofluids (suspension of magnetic nanoparticles in non-magnetic liquid media) present high Soret coefficients ${ }^{25,26}$ as well as an additional control parameter because of their magnetic nature and are thus considered to be promising candidates for such investigations. We have recently reported the combined experimental study on the thermodiffusion and Seebeck coefficient in ferrofluids ${ }^{27}$ where both Soret and Seebeck coefficients were measured as a function of nanoparticle (NP) concentration. While the resulting thermoelectric power was found to decrease by the inclusion of magnetic nanoparticles in a DMSO (dimethyl sulfoxide) based ferrofluid, our work has served as a proof-of-principle demonstrating the contribution of thermodiffusion of charged colloidal particles to the total thermoelectric voltage of the carrier fluid. More importantly, we have identified specific experimental parameters such as a particle's Eastman entropy of transfer, its surface charge and the thermodiffusive direction (thermophilic or thermophobic) that can be used to ultimately enhance the thermoelectric property of charged colloidal liquids.

Here we focus our attention on one such experimental parameter, namely, the electrolyte type and investigate its effect on the ferrofluids' Seebeck coefficient $\left(S_{\mathrm{e}}\right)$. More specifically, we examine two aqueous ferrofluids made of identical citrate-coated maghemite nanoparticles that are ionically stabilized with either $\mathrm{TBuA}^{+}$(hereafter referred to as FF-TBuA) or $\mathrm{Li}^{+}$(FF-Li) counterions. $\S$ As in our previous study, ${ }^{27} S_{\mathrm{e}}$ is measured as a function of magnetic nanoparticle concentration. We demonstrate that with appropriate control over concerned physical parameters, one can indeed increase the thermoelectric power of the thermocell. We show that the choice of counterions greatly influences the nanoparticle interactions with the surrounding fluids, leading to modifications in the overall fluid's electrical (electrostatic) and thermoelectric properties. In the case of FF-TBuA, the Seebeck coefficient is found to increase as much as 15 percent for a NP volume fraction of $\sim 1 \%$. Since the efficiency of a thermocell varies with $S_{\mathrm{e}}{ }^{2}$, the expected efficiency increase is as much as 30 percent. We show that the observed enhancement is likely due to: (1) a large nanoparticles' electrical conductivity and Eastman entropy of transfer and (2) an important Eastman entropy of transfer of certain counterions. Furthermore, the electrical conductivity of the overall ferrofluid also increases by the introduction of charged nanoparticles. Combined together, the findings reported here show a brand new possibility to improve the energy conversion capability of liquid thermoelectric materials using charged colloidal particles.

$\S$ These ferrofluids were previously measured for their thermodiffusion properties which revealed that the counterions control the magnitude and the sign of the nanoparticle Soret coefficients; i.e., a large and positive (thermophobic) value for FF-TBuA, while a smaller and negative (thermophilic) value for FF-Li. ${ }^{25}$
In the next paragraph, the theoretical framework concerning the production of thermoelectric potential in thermogalvanic cells is given. We then describe the experimental methods for thermoelectric measurements and ferrofluid preparation techniques. Last but not least we discuss the experimental results obtained for initial and apparent steady state $S_{\mathrm{e}}$ values in light of numerical calculations.

\section{General theory}

When a temperature gradient is applied to a thermoelectric cell, one can distinguish between the initial state, when the concentrations are still homogeneous, just after the temperature gradient has been imposed, and the Soret equilibrium state characterized by a zero-flux condition for all species in the system.

\subsection{Initial state}

At the onset of a thermal gradient application, a thermocell consisting of an electrolyte with a redox couple and charged colloidal particles produces an open circuit voltage from two sources. The corresponding Seebeck coefficient is expressed as:

$$
S_{\mathrm{e}}^{\mathrm{ini}}=\frac{1}{e}[\underbrace{-\Delta \boldsymbol{s}_{\mathrm{rc}}}_{\text {electrodes' surface }}+\overbrace{\sum_{i} \frac{t_{i} \hat{S}_{i}}{\xi_{i}}}^{\text {bulk }}]
$$

The first term describes the thermogalvanic potential, $\Delta \boldsymbol{s}_{\mathrm{rc}} / e$, generated by the temperature dependent reaction entropy of redox couples at the electrode surface. $\Delta \boldsymbol{s}_{\mathrm{rc}}$ is expressed by the Nernst equation,

$$
\Delta s_{\mathrm{rc}}=\Delta s_{\mathrm{rc}}^{\circ}+\frac{k_{\mathrm{B}}}{n \cdot \Delta T}\left[T_{\mathrm{h}} \ln \left(\frac{a_{\mathrm{h}}^{\mathrm{ox}}}{a_{\mathrm{h}}^{\mathrm{red}}}\right)-T_{\mathrm{c}} \ln \left(\frac{a_{\mathrm{c}}^{\mathrm{ox}}}{a_{\mathrm{c}}^{\mathrm{red}}}\right)\right]
$$

where $\Delta s_{\mathrm{rc}}^{\circ}$ is the standard redox reaction entropy, $k_{\mathrm{B}}$ is the Boltzmann constant, $n$ is the number of electrons transferred in the redox reaction, and $\Delta T=T_{\mathrm{h}}-T_{\mathrm{c}}, T_{\mathrm{h}}$ and $T_{\mathrm{c}}$ being the temperature at the hot and cold electrodes, respectively. $a=\gamma \cdot c$ is the activity where $c$ is the molar concentration of the species and $\gamma$ is its activity coefficient that depends on the ionic strength of the solution. The activity coefficients for the ferro-/ferri-cyanide redox couple in water are well documented for a wide range of ionic strengths. ${ }^{28}$ As the ionic strength of a charged colloidal solution is known to depend on the presence of all ions (free salts and counterions surrounding the charged colloidal particles), ${ }^{29,30}$ the inclusion of charged nanoparticles (and their concentration) will modify the thermogalvanic contribution to the overall Seebeck coefficient.

The second term in eqn (1) is a thermodiffusion term. It stems from the initial electric field created in the bulk of the cell by the thermodiffusion of all charged species present in the solution. $t_{i}$ is the Hitthorf transport number, corresponding to the relative contributions of ionic conductivities $\sigma_{i}$ to the total conductivity: $t_{i}=\sigma_{i} / \sum_{i} \sigma_{i}$. 
$\hat{S}_{i}$ is the Eastman entropy of transfer of $i$ th charged species. For small point-like ions, the dimensionless number $\xi_{i}$ is simply the ionic charge number $z_{i}$. For colloidal particles, which are macro-ions, $\xi_{i}$ is the dynamic effective charge.

The volume fraction $\phi$ dependence of the nanoparticles' $\hat{S}$ and $\xi$ in eqn (1) can be inferred from a hard-sphere model. In the Carnahan-Starling equation of state for a hard-sphere gas, the isothermal osmotic compressibility is given by:

$$
\chi\left(\phi_{\text {eff }}\right)=\frac{\left(1-\phi_{\text {eff }}\right)^{4}}{1+4 \phi_{\text {eff }}+4 \phi_{\text {eff }}{ }^{2}-4 \phi_{\mathrm{eff}^{3}}+\phi_{\mathrm{eff}^{4}}^{4}}
$$

where the effective volume fraction $\phi_{\mathrm{eff}}=\phi\left(d_{\mathrm{HC}} / d\right)^{3}$, with an effective hard core diameter $d_{\mathrm{HC}}=d+2 \lambda_{\mathrm{D}}$, where $\lambda_{\mathrm{D}}$ is the screening length. We obtain [cf. ref. 27]

$$
\hat{S} \approx \hat{S}_{0} \cdot \chi\left(\phi_{\text {eff }}\right) \text { and } \xi=\xi_{0} \cdot \chi\left(\phi_{\text {eff }}\right)
$$

where $\hat{S}_{0}$ and $\xi_{0}$ are defined as the limit $\phi \rightarrow 0$ of $\hat{S}$ and $\xi$ respectively. As can be seen from the equation above, the relative magnitude of $\hat{S}_{i}$ as well as the size and the sign of the effective charge of charged species (ions and charged particles) determines whether a positive or negative contribution is made to the total initial Seebeck coefficient.

\subsection{Soret equilibrium}

2.2.1 Seebeck coefficient at the electrodes. At the Soret equilibrium, the Seebeck coefficient measured at the electrodes is expressed as: ${ }^{17,27}$

$$
S_{\mathrm{e}}^{\mathrm{Eq}}=-\frac{\Delta V_{\text {electrodes }}}{\Delta T}=\frac{1}{e}\left[-\Delta \boldsymbol{s}_{\mathrm{rc}}+\sum_{j} \lambda_{j} \cdot \hat{S}_{j}\right]
$$

where $\Delta V_{\text {electrodes }}$ and $\Delta T$ are, respectively, the voltage and temperature differences between the two electrodes, the sum is over the ionic species $j$ participating in the redox reaction and $\lambda_{j}$ is defined by the redox chemical equation, for instance:

$$
\lambda_{\text {ox }} \cdot \mathrm{Ox}+\mathrm{e}^{-}+\lambda_{\text {red }} \cdot \operatorname{Red}=0
$$

Therefore, $S_{\mathrm{e}}^{\mathrm{Eq}}$ depends directly only on the species intervening in the redox reaction (see ref. 27 for more in-depth development). The ionic strength and consequently, the activity coefficient of the redox reaction potential is modified due to the inclusion of charged magnetic nanoparticles. However, this variation in the ferrofluids studied here remains a minor effect. ${ }^{31}$

2.2.2 Bulk Seebeck coefficient and the Soret effect. When the Soret equilibrium is established; i.e. no more diffusion occurs, the bulk distribution of charged nanoparticles under a given thermal gradient can be given by

$$
\frac{\nabla n}{n}=-\alpha(t) \nabla T ; \quad \alpha=\left(\hat{S}-\xi e S_{\mathrm{e}}^{\mathrm{Eq} *}\right) / k_{\mathrm{B}} T
$$

where $n$ is the nanoparticle concentration, $\alpha$ is the Soret coefficient and $S_{\mathrm{e}}^{\mathrm{Eq} *} \nabla T=E^{\mathrm{Eq}}$ corresponds to the thermoelectric field in the bulk of the solution, whose derived potential is different from the thermoelectric potential measured at the electrodes. Under a thermal gradient, nanoparticles accumulate near one electrode and deplete from the other, thus creating unequal ionic concentrations at each ends, affecting the overall temperature dependence of the reaction entropy of redox couples.

\section{Experimental}

\subsection{Aqueous ferrofluid samples}

The two aqueous ferrofluids used in the present study are based on maghemite $\left(\gamma-\mathrm{Fe}_{2} \mathrm{O}_{3}\right)$ nanoparticles with an average diameter of $7.6 \mathrm{~nm}$, synthesized following the method developed by Massart. $^{32}$ The nanoparticles are citrate-coated (with citrate co-ions) and electrostatically stabilized with monovalent counterions of lithium (FF-Li) or tetrabutyl ammonium (FF-TBuA) in water at $\mathrm{pH}$ 7. The NPs have a negative structural charge $Z_{\text {str }}$

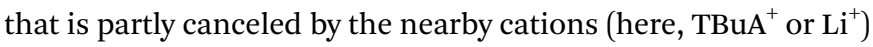
condensed within the NPs' first solvation layers, resulting in a much smaller dynamic effective charge $\xi_{0}$. The concentrations of free co-ions (citrate, $30 \mathrm{mM}$ ) and counterions $\left(\mathrm{Li}^{+}\right.$and $\mathrm{TBuA}^{+}$, $90 \mathrm{mM}$ ) are determined via electrical conductivity measurements performed on the supernatant solutions obtained after ultracentrifugation of concentrated ferrofluids. For the present Seebeck coefficient measurements, $3 \mathrm{mM}$ ferro-/ferricyanide redox couple was added to the ferrofluids. All free-ion concentrations were kept constant throughout the subsequent measurements. The characteristics of ferrofluids FF-Li and FF-TBuA are summarized in Table 1. For more detailed information on the synthesis method and the ion concentration measurements, please refer to ref. 25 .

\subsection{Seebeck coefficient and power measurements}

The thermoelectric voltage measurements were carried out in a single-volume vertical cell with a $6 \mathrm{~mm}$ diameter sample cavity machined out from a solid Teflon cylinder closed by two symmetrical $10 \mathrm{~mm}$ diameter platinum foil electrodes of $100 \mu \mathrm{m}$ thickness (99.99\% pure, Alfa Aesar) (see Fig. 1(a)). The Pt electrode surface was cleaned by immersion in a concentrated $\mathrm{HCl}$ (36 wt\%) solution and subsequently by ultrasonication in ultrapure water. The electrode surface in contact with the liquid is $A \approx 0.28 \mathrm{~cm}^{2}$ and the distance between the electrodes is $l=6 \mathrm{~mm}$. Two $10 \mathrm{~mm}$ thick copper blocks are screwed onto the cell body, thereby squeezing the Pt electrodes hermetically against the Teflon cylinder (the sample volume is $0.17 \mathrm{~cm}^{3}$ ). The cell was always heated from the top to avoid natural convection in the fluid. The experiments are carried out between $25{ }^{\circ} \mathrm{C}$ and $55{ }^{\circ} \mathrm{C}$ with the temperature difference between the two electrodes being 0 , 10,20 or $30 \mathrm{~K}$. The temperature gradient along the vertical axis was stabilized within several minutes.

Table 1 Ferrofluid composition and parameters. $d$ is the average structural diameter and $C_{\mathrm{ci}}$ and $C_{\mathrm{rx}}$ are the counterion and redox concentrations. Ferro-/ferri-cyanide $\mathrm{Fe}(\mathrm{CN})_{6}{ }^{3-} / \mathrm{Fe}(\mathrm{CN})_{6}{ }^{4-}$ redox couple was introduced as salts of $\mathrm{Fe}(\mathrm{CN})_{6} \mathrm{~K}_{3}(\geq 99.98 \%)$ and $\mathrm{Fe}(\mathrm{CN})_{6} \mathrm{~K}_{4} \cdot 3 \mathrm{H}_{2} \mathrm{O}(\geq 99.95 \%$, both purchased from Sigma Aldrich and used as received)

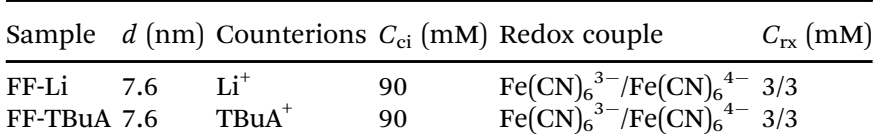




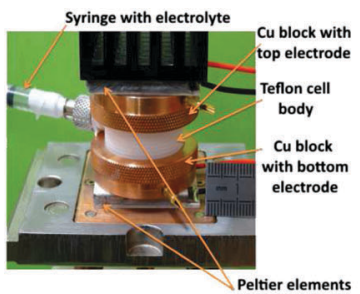

(a)
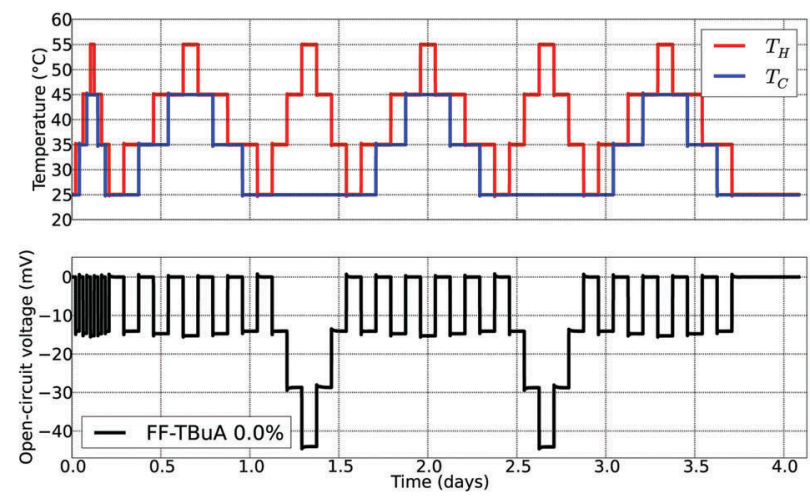

(c)
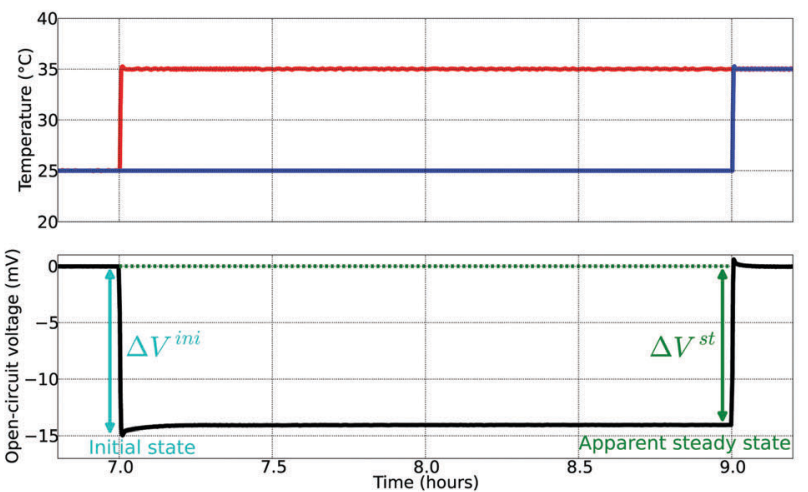

(d)

Fig. 1 (a) Thermocell, (b) thermocell schematic, and (c) typical thermoelectric measurements. $T_{H}$ is the hot electrode temperature and $T_{C}$ is the cold electrode temperature. The sample is FF-TBuA at $\phi=0 \%$. (d) Zoom-in view of one of the steps with $T_{\text {mean }}=25^{\circ} \mathrm{C}$. An apparent steady state is reached after a few hours. See text for more details.

The open circuit voltage between the two electrodes, $\Delta V$, was measured using a high impedance electrometer (Keithley 6514) and the Seebeck coefficient $S_{\mathrm{e}}$ was determined via $\Delta V=-S_{\mathrm{e}} \Delta T$. The thermoelectric voltage was monitored over 8 to 10 hours between each temperature change for $\phi>0$. This time was sufficient to extract the initial state Seebeck coefficient, $S_{\mathrm{e}}^{\mathrm{ini}}$, and to reach an apparent steady state, i.e. $S_{\mathrm{e}}$ reaches a constant value, $S_{\mathrm{e}}^{\text {st }}$ (see Fig. 1(d)), which is distinguished from $S_{\mathrm{e}}^{\mathrm{Eq}}$ described in eqn (5) for reasons discussed in the Results and discussion section. Both $S_{\mathrm{e}}^{\mathrm{ini}}$ and $S_{\mathrm{e}}^{\text {st }}$ are measured for all ferrofluid concentrations, and the measurements are quite reproducible over several weeks with data dispersion less than $4 \%$ (see Fig. 1(c)).

The same cell was used as well for AC electrical conductivity and power measurements. The conductivity measurements presented in Fig. 2(b) inset were carried out using a precision LCR meter (HP 4284A) at $150 \mathrm{kHz}$ which is the frequency where the imaginary part of the impedance is canceled. For power measurements, the cell, below $\Delta T=30 \mathrm{~K}$, was connected to a variable discharge resistor $R$ ranging from $1 \Omega$ to $10 \mathrm{M} \Omega$. After a transient regime corresponding to the reaction of the redox couple in the vicinity of the electrodes, the system reached an apparent steady state. The current was deduced from the steady state voltage using Ohm's law and the power was expressed as $P(R)=\Delta V_{\text {st }}^{2} / R$.

\section{Results and discussion}

The normalized initial and apparent steady state Seebeck coefficients, $S_{\mathrm{e}}^{\mathrm{ini}}$ and $S_{\mathrm{e}}^{\mathrm{st}}$, as a function of nanoparticle volume fraction $(\phi)$ are shown in Fig. 2 and 3 for FF-TBuA and FF-Li. The measurements were performed at mean temperatures of 30,40 and $50{ }^{\circ} \mathrm{C}$ with a constant $\Delta T$ value of $10 \mathrm{~K}$.

In Fig. 2(a) and (b), we can see that $S_{\mathrm{e}}^{\text {ini }}$ remains nearly constant as a function of $\phi$ for FF-Li whereas it increases by 10 to $15 \%$ for FF-TBuA. Fig. 3(a) shows the variation of $S_{\mathrm{e}}^{\text {st }}$ as a function of $\phi$ for both FF-Li and FF-TBuA. We can observe that, again, $S_{\mathrm{e}}^{\mathrm{st}}$ remains nearly constant up to $1 \%$ for FF-Li. On the other hand, for FF-TBuA the variation is quite peculiar with a rapid decrease down to a minimum reached at $\phi=0.1 \%$ followed by a linear increase. Last but not least, Fig. 3(b) presents the normalized difference between $S_{\mathrm{e}}^{\mathrm{ini}}$ and $S_{\mathrm{e}}^{\mathrm{st}}$ for FF-TBuA. This difference grows quickly until $\phi=0.1 \%$ and then remains constant in the concentration range studied, implying that the slopes of both $S_{\mathrm{e}}^{\text {ini }}(\phi)$ (Fig. 2(b)) and $S_{\mathrm{e}}^{\text {st }}(\phi)$ (Fig. 3(a), red curve) are identical for $\phi>0.1 \%$.

\subsection{Validation of measurements at $\phi=0 \%$}

At $\phi=0 \%, S_{\mathrm{e}}^{\mathrm{ini}}$ values were positive, as expected from the redox reaction entropy of the ferrocyanide/ferricyanide couple ${ }^{33}$ and agrees well with reported values in the literature; i.e., $1.48 \mathrm{mV} \mathrm{K}^{-1}$ at $30{ }^{\circ} \mathrm{C}$ close to a value of $1.43 \mathrm{mV} \mathrm{K}^{-1}$ reported in ref. 23 . The difference between $S_{\mathrm{e}}^{\mathrm{ini}}$ and $S_{\mathrm{e}}^{\text {st }}$ of $50 \pm 20 \mu \mathrm{V} \mathrm{K}^{-1}$ for FF-TBuA, as can be calculated from the values indicated in Fig. 2(b) $\left(S_{\mathrm{e}}^{\mathrm{ini}}\right)$ and Fig. 3(a) $\left(S_{\mathrm{e}}^{\mathrm{st}}\right)$, coincides well with the theoretically expected value of $60 \mu \mathrm{V} \mathrm{K}^{-1}$ calculated by summing the thermodiffusion term of all ions, i.e., redox species and solute ions, (see eqn (1)) and subtracting the second term of eqn (5). These observations confirm that the measured Seebeck coefficient values are indeed a combination of thermogalvanic and thermodiffusion effects measured in the initial and steady states of the diffusion process.

\subsection{Initial Seebeck coefficient as a function of $\phi$}

As can be seen from Fig. 2(a) and (b), the initial Seebeck coefficient remains nearly constant when $\phi$ increases up to $1 \%$ (within experimental error bars) for FF-Li whereas for FF-TBuA an increase between 10 and 15\% is observed.

The initial Seebeck coefficient, as defined by eqn (1), contains both thermogalvanic and thermodiffusion terms. As explained 


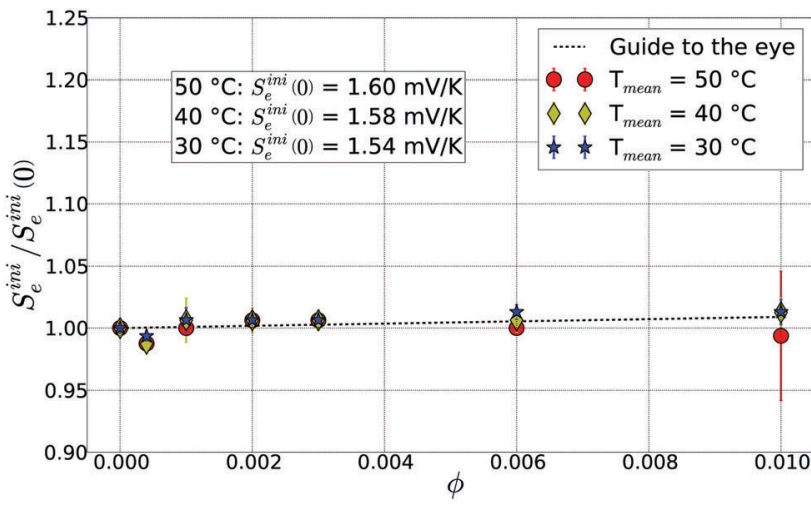

(a)

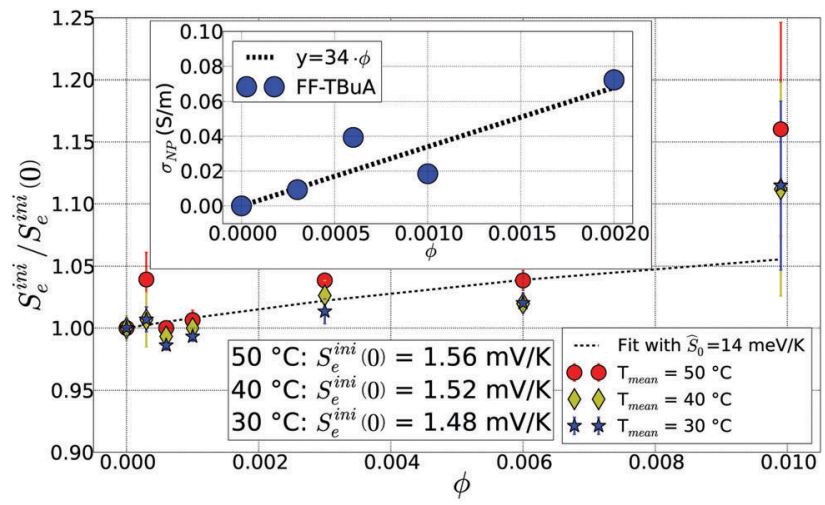

(b)

Fig. 2 (a) Normalized initial state Seebeck coefficient as a function of the NP volume fraction $(\phi)$ for FF-Li, (b) normalized initial state Seebeck coefficient and NP electrical conductivity (inset) as a function of $\phi$ for FF-TBuA. $S_{e}(0)$ is the value measured without nanoparticles. $\Delta T=10 \mathrm{~K}$ for both figures. The error bars correspond to twice the standard error (95\% confidence interval).

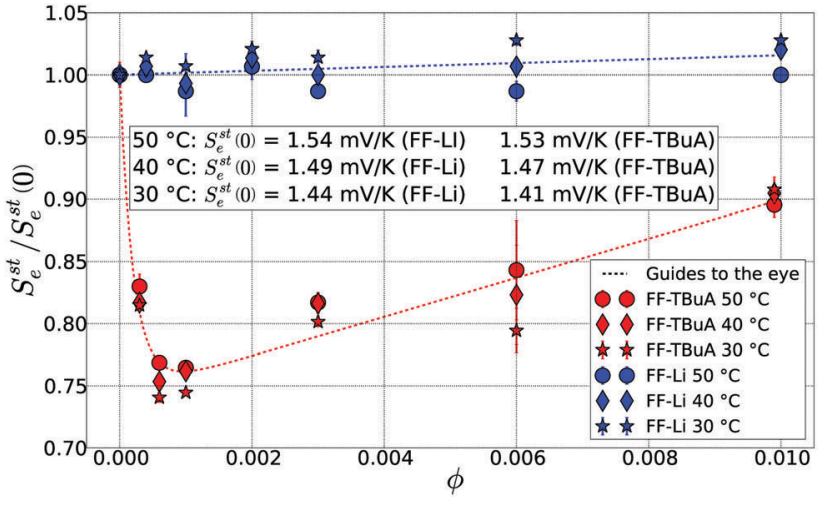

(a)

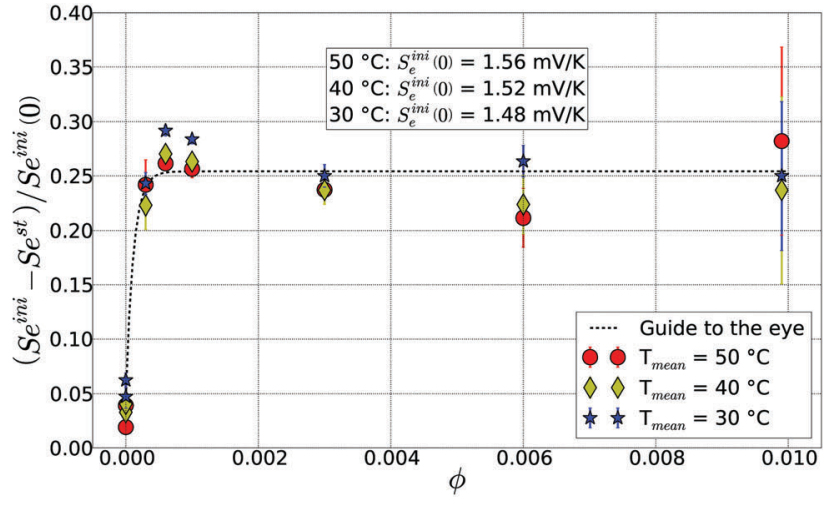

(b)

Fig. 3 (a) Normalized steady state Seebeck coefficient as a function of $\phi$ for both FF-Li and FF-TBuA, (b) normalized difference between the initial and steady state Seebeck coefficients as a function of $\phi$ for FF-TBuA. $S_{e}(0)$ is the value measured without nanoparticles. $\Delta T=10 \mathrm{~K}$ for both figures. The error bars correspond to twice the standard error (95\% confidence interval).

earlier both of these terms depend on the nanoparticle concentration, however, the distribution of different species (nanoparticles, counterions, salts and redox couples) is still uniform $^{17}$ at this stage. In order to calculate the thermogalvanic contribution using eqn (2), one needs to know the redox species activity coefficient whose values are tabulated as a function of ionic strength. ${ }^{28}$ The total ionic strength $I_{\text {tot }}$ of a charged colloidal solution with monovalent counterions (such as $\mathrm{TBuA}^{+}$ and $\mathrm{Li}^{+}$used in this study) is given by ${ }^{30}$

$$
I_{\mathrm{tot}}=\sum_{i \neq \mathrm{NP}} \frac{z_{i}^{2} \cdot c_{i}}{2}=I+\frac{Z_{\mathrm{eff}} \cdot n}{2 \cdot N_{\mathrm{A}}}
$$

where $I$ is the ionic strength produced by solute ions of molar concentration $c_{i}$; i.e., $\mathrm{TBuA}^{+}, \mathrm{Cit}^{3-}$ (assimilated here to 3 independent acetate ions - see ESI $1 \dagger$ and ref. 25) and redox couple species. The second term comes from the electroneutrality of the solution with $Z_{\text {eff }}$ being the effective charge of nanoparticles and $n$ the NP concentration.
Assuming the uniform distribution of nanoparticles at the electrode surfaces to be the same as that in the bulk, the change in the thermogalvanic coefficient due to the inclusion of charged magnetic nanoparticles is calculated to be less than $5 \mu \mathrm{V} \mathrm{K}^{-1}$. This is much smaller than the observed change in the initial state Seebeck coefficient $\left(180 \mu \mathrm{V} \mathrm{K}{ }^{-1}\right)$ as well as the error bars of our measurements $\left(\sim 10 \mu \mathrm{V} \mathrm{K} \mathrm{K}^{-1}\right)$, suggesting that the observed increase stems primarily from the second term in eqn (1).

As the nanoparticle concentration $(n)$ is increased, the number of $\mathrm{TBuA}^{+}$ions increases linearly, while citrate and redox couple molecule concentrations $(3 \mathrm{mM})$ remain constant. Thus, the Hittorf number of all charged species varies somewhat and most importantly for $\mathrm{TBuA}^{+}$and nanoparticles, and the second term in eqn (1) is also modified.

Using the measured electrical conductivity of ferrofluid samples, the known values of ionic conductivity and the Eastman entropy of transfer of ions found in the literature, ${ }^{17,34}$ one can fit the data for Fig. 2(b) to eqn (1) to obtain the Eastman entropy of 
transfer of nanoparticles. Since $\hat{S}$ and $\xi$ depend similarly on $\chi\left(\phi_{\text {eff }}\right)$, then eqn (1) becomes:

$$
S_{\mathrm{e}}^{\mathrm{ini}}(\phi)=\frac{1}{e}\left[-\Delta \boldsymbol{s}_{\mathrm{rc}}+\sum_{i \neq \mathrm{NP}} \frac{t_{i}(\phi) \hat{S}_{i}}{\xi_{i}}+\frac{t_{\mathrm{NP}}(\phi) \hat{S}_{0}}{\xi_{0}}\right]
$$

Here, $t_{\mathrm{NP}}$ and $\xi_{0}$ are experimentally determined. $\hat{S}_{0}$ of the nanoparticles, determined from the best fit of the experimental data, $\uparrow$ is $14 \mathrm{meV} \mathrm{K}^{-1}$ for FF-TBuA, slightly smaller in magnitude than that found in our previous study using DMSO-based ferrofluids (see ref. 27 and ESI $2 \dagger$ ). However, this value must be considered with caution due to a large experimental uncertainty in the measured values of electrical conductivity which were measured only at low NP concentrations.

In the case of FF-Li, we do not see a similar change in $S_{\mathrm{e}}^{\mathrm{ini}}$ and thus cannot draw any quantitative conclusion. However we can propose a few possible explanations: $(1) \hat{S}_{0}$ is very small as well as $\xi_{0}$ (note that, to a first approximation, $S_{\mathrm{e}}^{\text {ini }}(\phi)$ grows with $\xi_{0}$ since $t_{\mathrm{NP}}$ is proportional to $\xi_{0}^{2}$ and therefore $\left.t_{\mathrm{NP}} \cdot \hat{S}_{0} / \xi_{0} \propto \xi_{0} \cdot \hat{S}_{0}\right)$ or (2) the second and third terms in eqn (9) happen to cancel each other.

\subsection{Stationary Seebeck coefficient}

The evolution of apparent steady state Seebeck coefficient behavior raises several questions. First, the apparent steady state is reached 6-8 hours after the temperature difference has been applied. However, considering the diffusion coefficient of nanoparticles in water $\left(D_{\mathrm{NP}} \sim 10^{-11} \mathrm{~m}^{2} \mathrm{~s}^{-1}\right)$ and the characteristic length $l \sim 6 \mathrm{~mm}$, one would expect the FF to reach its true Soret steady state in $\tau=l^{2} /\left(\pi^{2} D_{\mathrm{NP}}\right) \sim 100$ hours. Numerical simulations using Mathematica of the time dependent NP concentration were computed at 3 different locations in the cell; i.e., at hot and cold ends and at mid-height, as shown in Fig. 4 . The particle current density $J_{\mathrm{NP}}$ is calculated using the diffusion equation:

$$
J_{\mathrm{NP}}=-D_{\mathrm{NP}}\left(n_{\mathrm{NP}}, t\right) \cdot\left[\nabla n_{\mathrm{NP}}+\alpha\left(n_{\mathrm{NP}}, t\right) \cdot n_{\mathrm{NP}} \nabla T\right]
$$

A temperature difference $\Delta T=10 \mathrm{~K}$ is applied at $t=0$ and two homogeneous NP concentrations of 1 and $0.4 \%$ are considered. Zero-flux boundary conditions are imposed at the borders of the cell (top and bottom). As can be seen from Fig. 4, after the time elapse of $6-8$ hours (i.e., $t \approx 0.1 \cdot \tau$ ), the NP distribution in the bulk is much closer to that in the initial state than in the Soret equilibrium one. This makes us speculate that the experimentally determined stationary state Seebeck $S_{\mathrm{e}}^{\text {st }}$ is not equal to the Soret equilibrium Seebeck $S_{\mathrm{e}}^{\mathrm{Eq}}$ as described in eqn (5). Similar simulation results were obtained by Putnam et al. ${ }^{36}$ for colloidal solutions (not ferrofluids) with a comparable Soret coefficient. Moreover, in thermodiffusion measurements on ferrofluids with a comparable experimental parameter (in terms of cell size, fluid and particle characteristics), nanoparticles are found to continue to thermodiffuse over several days and longer. ${ }^{37}$

T In order to account for the experimentally measured increase in electrical conductivity, the dynamic effective charge $\xi_{0}$ of the FF-TBuA nanoparticles had to be around $-300 e$. This value was used in the fit shown in Fig. 2(b) and for the numerical simulations presented in Fig. 4, and is comparable to the dynamic electrical charge that has been measured in ref. 35 by electrical conductivity.

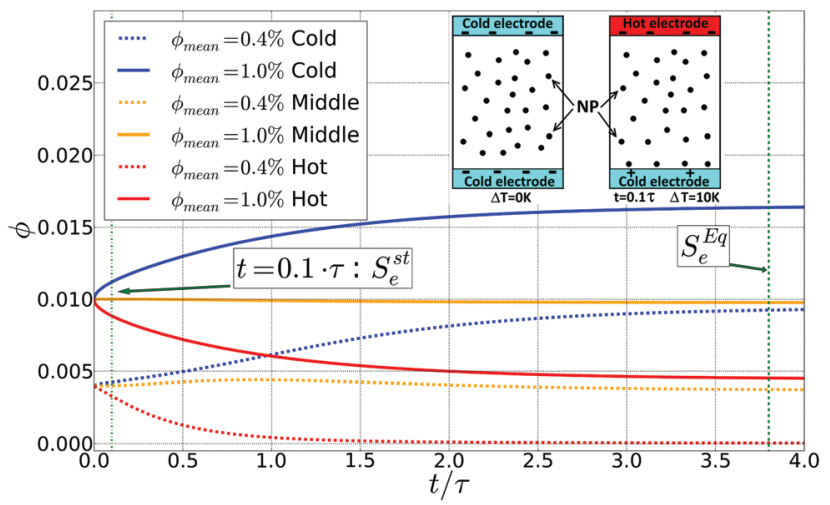

Fig. 4 Analytic simulation of the nanoparticle concentration as a function of $\tau$ calculated through eqn (10). At $t=0$, the temperature difference $\Delta T=10 \mathrm{~K}$ is imposed upon two FF-TBuA solutions of $1 \%$ and $0.4 \%$ starting volume fractions. The solid and dotted blue lines are the volume fractions at the cold electrode, the orange lines are the volume fractions in the middle of the cell and the red lines are the volume fractions at the hot electrode. The solid lines correspond to $1 \%$ starting volume fraction and the dotted lines to $0.4 \%$ starting volume fraction. These simulations consider only the bulk diffusion and do not account for specific effects happening at the electrode surfaces. A dynamic effective charge $\xi_{0}$ of -300 was used for these simulations.

Unexplained is then the minimum appearing in $S_{\mathrm{e}}^{\mathrm{st}}$ at $\phi=0.1 \%$ for FF-TBuA (Fig. 3(a)). A closer examination of experimental data reveals that the increase in $S_{\mathrm{e}}^{\text {st }}(\phi)$ for $\phi>0.1 \%$ follows the same trend as the increase found in $S_{\mathrm{e}}^{\mathrm{ini}}(\phi)$. To illustrate this point, we have plotted $\Delta S_{\mathrm{e}}=S_{\mathrm{e}}^{\text {ini }}-S_{\mathrm{e}}^{\text {st }}$ in Fig. 3(b). Knowing that at the time of $S_{\mathrm{e}}^{\text {st }}$ the ferrofluids are still in their initial state, the subtraction of $S_{\mathrm{e}}^{\text {st }}$ from $S_{\mathrm{e}}^{\mathrm{ini}}$ cancels the thermodiffusion term in eqn (1). Therefore, the $\Delta S_{\mathrm{e}}$ evolution is due to an additional effect on the thermogalvanic reaction potential $\left(\Delta s_{\mathrm{rc}}\right.$ in eqn (1)) taking place strictly at the electrodes' surface. This effect increases rapidly at very low values of $\phi$ and saturates beyond a critical concentration value of $\phi=0.1 \%$. Furthermore, this effect is reversible (Fig. 1(c)).

According to eqn (2) the thermogalvanic term $\Delta \boldsymbol{s}_{\mathrm{rc}}$ consists of $\Delta s_{\mathrm{rc}}^{\circ}$ corresponding to the standard partial entropy and the Nernst term that depends on the molar concentration ratios of the redox couple species and their activity coefficients at the hot and cold electrodes. It is known that $\Delta s_{\mathrm{rc}}^{\circ}$ is strongly dependent on the redox couple molecular environment (e.g., solvent's dielectric constant or acceptor number); ${ }^{38}$ which can explain the large disparity in the reported values of thermogalvanic coefficient of aqueous ferro-/ferri-cyanide redox couple with Pt electrodes. ${ }^{39}$ Therefore both components of $\Delta \boldsymbol{s}_{\mathrm{rc}}$ depend on the local ionic strength and thus are influenced by the nanoparticles' concentration difference at the hot and cold electrode surfaces.

Our present hypothesis is that as a voltage is created between the two electrodes due to the temperature gradient, one electrode charge becomes less negative than the other one, allowing the nanoparticles to approach its surface. This effect, which takes 6-8 hours to settle in, influences the redox couple ionic environment and thus $\Delta s_{\mathrm{rc}}$. However, it saturates quickly 


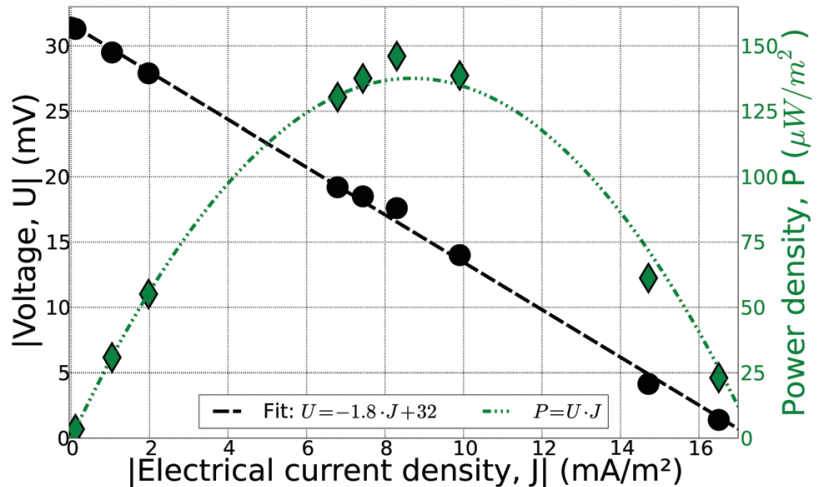

Fig. 5 Power measurements for FF-TBuA $0.06 \%$ with a temperature difference of $30 \mathrm{~K}$ between the top and bottom electrodes. The cell is discharged on a variable resistor ranging from $1 \Omega$ to $10 \mathrm{M} \Omega$.

as the electrostatic repulsion created by the already adsorbed nanoparticles prevents additional particles from approaching, resulting in a plateau for $\phi>0.1 \%$. In a ferrofluid similar to ours, nanoparticles adsorption on mercury and gold electrodes has been observed in ref. 40, lending support to our hypothesis. Nanoparticle adsorption measurements using an in situ quartz crystal microbalance at different applied electric potentials are underway to verify this scenario.

A natural question may be, then, why FF-Li does not show ionic strength change effect on the apparent stationary Seebeck coefficient. The answer to this question is not clear, however, it could be partially due to a lower surface charge of Li-stabilized nanoparticles.

Lastly, as a proof of concept, we have measured the power output of FF-TBuA for a concentration of $0.06 \%$ (see Fig. 5). The current and voltage measured as described in the Experimental section are stable over several hours. As expected, a linear relation is found between the voltage and the current density. The largest power density is $\sim 150 \mu \mathrm{W} \mathrm{m}{ }^{-2}$, comparable to values found in the literature. ${ }^{41}$

\section{Conclusion}

Aqueous ferrofluids containing ionically stabilized colloidal magnetic nanoparticles and the ferro-/ferri-cyanide redox couple were used as alternatives to conventional electrolytes for liquid thermoelectric cells. Both thermogalvanic and thermodiffusion contributions to the fluids' Seebeck coefficient were found to depend on the nanoparticle concentration and electrolyte $\left(\mathrm{Li}^{+}\right.$and $\left.\mathrm{TBuA}^{+}\right)$. An additional (thermo)electric effect, most likely due to the electrostatic adsorption of nanoparticles on the electrodes' surface, was witnessed. This influences the apparent steady state Seebeck coefficient at low nanoparticle concentrations. For ferrofluids containing $\mathrm{TBuA}^{+}$as counterions, the inclusion of magnetic nanoparticles leads to an enhancement of the fluid's initial Seebeck coefficient by $15 \%$ (at nanoparticle concentration $\sim 1 \%$ ). Since, to a first approximation, the efficiency of a thermocell is proportional to the figure of merit $Z T=\frac{S e^{2} \sigma T}{\kappa}(\sigma$ and $\kappa$ are, respectively, the electrical and thermal conductivities) and therefore to the Seebeck coefficient squared, the increase of the expected efficiency is $30 \%$. One should note that for a liquid thermocell, the electrical conductivity to be considered in $Z T$ is that of the redox couple at a low frequency, rather than the conductivity of the liquid itself. Within the existing theoretical framework, the observed increase can be attributed to the large value of Eastman entropy of transfer and possibly a large effective surface charge of colloidal particles. The corollary of this statement sets a direction for future thermoelectric research in colloidal solutions. These findings open a new technological path for improving the efficiency of liquid thermoelectrochemical flow cells using charged colloidal particles where the system is always in the initial state.

\section{Acknowledgements}

This work was supported by ANR TEFLIC (Grant No. ANR-12PRGE-0011-01) and LABEX-PALM (Grant No. ANR-10-LABX-0039PALM). C. Lopes Filomeno is very grateful for a CAPES grant no. 99999.001111/2014-00 and for CAIQ/UnB support.

\section{References}

1 L.-D. Zhao, G. Tan, S. Hao, J. He, Y. Pei, H. Chi, H. Wang, S. Gong, H. Xu, V. P. Dravid, C. Uher, G. J. Snyder, C. Wolverton and M. G. Kanatzidis, Science, 2016, 351, 141-144.

2 Z. Chen, G. Han, L. Yang, L. Cheng and J. Zou, Prog. Nat. Sci.: Mater. Int., 2012, 22, 535-549.

3 M. Elsheikh, D. Shnawah, M. Sabri, S. Said, M. Hassan, M. Bashir and M. Mohamad, Renewable Sustainable Energy Rev., 2014, 30, 337-355.

4 H. Alam and S. Ramakrishna, Nano Energy, 2013, 2, 190-212.

5 V. Andrei, K. Bethke and K. Rademann, Energy Environ. Sci., 2016, 9, 1528-1532.

6 A. Gunawan, C. Lin, D. Buttry, V. Mujica, R. Taylor, R. Prasher and P. Phelan, Nanoscale Microscale Thermophys. Eng., 2013, 17, 304-323.

7 S. Uhl, E. Laux, T. Journot, L. Jeandupeux, J. Charmet and H. Keppner, J. Electron. Mater., 2014, 43, 3758-3764.

8 M. A. Lazar, D. Al-Masri, D. R. MacFarlane and J. M. Pringle, Phys. Chem. Chem. Phys., 2016, 18, 1404-1410.

9 D. R. MacFarlane, N. Tachikawa, M. Forsyth, J. M. Pringle, P. C. Howlett, G. D. Elliott, J. H. Davis, M. Watanabe, P. Simon and C. A. Angell, Energy Environ. Sci., 2014, 7, 232-250.

10 T. Abraham, D. MacFarlane, R. Baughman, L. Jin, N. Li and J. Pringle, Electrochim. Acta, 2013, 113, 87-93.

11 T. Abraham, D. MacFarlane and J. Pringle, Energy Environ. Sci., 2013, 6, 2639-2645.

12 K. Sandbakk, A. Bentien and S. Kjelstrup, J. Membr. Sci., 2013, 434, 10-17. 
13 A. Gunawan, H. Li, C. Lin, D. Buttry, V. Mujica, R. Taylor, R. Prasher and P. Phelan, Int. J. Heat Mass Transfer, 2014, 78, 423-434.

14 L. Zhang, T. Kim, N. Li, T. J. Kang, J. Chen, J. M. Pringle, M. Zhang, A. H. Kazim, S. Fang and C. Haines, et al., Adv. Mater., 2017, 1605652.

15 E. D. Eastman, J. Am. Chem. Soc., 1928, 50, 283-291.

16 S. De Groot, J. Phys. Radium, 1947, 8, 193-200.

17 J. Agar, in Thermogalvanic Cells, ed. P. Delahay, Interscience, New York, 1963, pp. 31-121.

18 A. Würger, Langmuir, 2009, 25, 6696-6701.

19 H. Ning, S. Datta, T. Sottmann and S. Wiegand, J. Phys. Chem. B, 2008, 112, 10927-10934.

20 S. Duhr and D. Braun, Proc. Natl. Acad. Sci. U. S. A., 2006, 103, 19678-19682.

21 D. Stadelmaier and W. Köhler, Macromolecules, 2009, 42, 9147-9152.

22 R. Piazza and A. Parola, J. Phys.: Condens. Matter, 2008, 20, 153102.

23 T. J. Kang, S. Fang, M. E. Kozlov, C. S. Haines, N. Li, Y. H. Kim, Y. Chen and R. H. Baughman, Adv. Funct. Mater., 2012, 22, 477-489.

24 H. Im, T. Kim, H. Song, J. Choi, J. S. Park, R. Ovalle-Robles, H. D. Yang, K. D. Kihm, R. H. Baughman and H. H. Lee, et al., Nat. Commun., 2016, 7, 10600.

25 C. L. Filomeno, M. Kouyaté, F. Cousin, G. Demouchy, E. Dubois, L. Michot, G. Mériguet, R. Perzynski, V. Peyre, J. Sirieix-Plénet and F. A. Tourinho, J. Magn. Magn. Mater., 2017, DOI: 10.1016/j.jmmm.2016.07.050.

26 T. Voelker and S. Odenbach, Phys. Fluids, 2005, 17, 037104.

27 B. T. Huang, M. Roger, M. Bonetti, T. J. Salez, C. WiertelGasquet, E. Dubois, R. Cabreira Gomes, G. Demouchy, G. Mériguet, V. Peyre, M. Kouyaté, C. L. Filomeno, J. Depeyrot, F. A. Tourinho, R. Perzynski and S. Nakamae, J. Chem. Phys., 2015, 143, 054902.
28 P. Vanysek, Activity Coefficients of Acids, Bases, and Salts, Handbook of Chemistry and Physics, CRC Press, 2014, ch. Section 5: Thermochemistry, Electrochemistry, and Solution Chemistry, pp. 5-104-5-105.

29 S. Grandner, Y. Zeng, R. v. Klitzing and S. H. L. Klapp, J. Chem. Phys., 2009, 131, 154702.

30 Y. Zeng, S. Grandner, C. L. P. Oliveira, A. F. Thunemann, O. Paris, J. S. Pedersen, S. H. L. Klapp and R. von Klitzing, Soft Matter, 2011, 7, 10899-10909.

31 The effective ionic strength of charged colloidal solutions (eqn (8)) was calculated using the steady state NP distribution found in Fig. 4. The variation in $\Delta \boldsymbol{s}_{\mathrm{rc}}$ due to the ionic strength difference is only of the order of $0.05 \mu \mathrm{V} \mathrm{K} \mathrm{K}^{-1}$, less than $0.02 \%$ of the measured change in $S_{\mathrm{e}}^{\mathrm{st}}$, and it cannot reproduce the observed minimum.

32 R. Massart, IEEE Trans. Magn., 1981, 17, 1247-1250.

33 J. Hupp and M. J. Weaver, Inorg. Chem., 1984, 23, 3639-3644.

$34 \mathrm{P}$. Vanysek, Ionic conductivity and diffusion at infinite dilution, Handbook of Chemistry and Physics, CRC Press, 2014, Section 5: Thermochemistry, Electrochemistry, and Solution Chemistry, pp. 5-77-5-79.

35 I. Lucas, S. Durand-Vidal, O. Bernard, V. Dahirel, E. Dubois, J. Dufrêche, S. Gourdin-Bertin, M. Jardat, G. Mériguet and G. Roger, Mol. Phys., 2014, 112, 1463-1471.

36 S. Putnam and D. Cahill, Langmuir, 2005, 21, 5317-5323.

37 L. Sprenger, A. Lange and S. Odenbach, Phys. Fluids, 2014, 26, 022001.

38 J. T. Hupp and M. J. Weaver, Inorg. Chem., 1984, 23, 3639-3644.

39 T. I. Quickenden and Y. Mua, J. Electrochem. Soc., 1995, 142, 3985-3994.

40 I. T. Lucas, E. Dubois, J. Chevalet and S. Durand-Vidal, Phys. Chem. Chem. Phys., 2008, 10, 3263-3273.

41 A. Gunawan, C.-H. Lin, D. A. Buttry, V. Mujica, R. A. Taylor, R. S. Prasher and P. E. Phelan, Nanoscale Microscale Thermophys. Eng., 2013, 17, 304-323. 